\title{
Kernos
}

Revue internationale et pluridisciplinaire de religion grecque antique

5 | 1992

Varia

\section{Le signe d'extase et la musique}

\section{Pierre Somville}

Édition électronique
URL: http://journals.openedition.org/kernos/1059

DOI : 10.4000/kernos.1059

ISSN : 2034-7871

\section{Éditeur}

Centre international d'étude de la religion grecque antique

\section{Édition imprimée}

Date de publication : 1 janvier 1992

ISSN : 0776-3824

\section{Référence électronique}

Pierre Somville, «Le signe d'extase et la musique », Kernos [En ligne], 5 | 1992, mis en ligne le 19 avril 2011, consulté le 20 avril 2019. URL : http://journals.openedition.org/kernos/1059; DOI : 10.4000/ kernos. 1059 
Kernos, 5 (1992), p. 173-181.

\section{LE SIGNE D'EXTASE ET LA MUSIQUE}

Ils jouaient la sonate à Kreutzer.

Connaissez-vous le premier presto?

Ah ! Quelle chose terrible...

TOLSTOİ

Je livre ici le bilan provisoire d'une recherche en cours sur un problème précis d'iconographie. Il s'agit du geste de ces symposiastes inclinant le buste vers l'arrière, se portant la main droite à la tête et se prenant le front, en y appliquant la paume ou l'avant-bras.

Ce mouvement, relativement fréquent, est bien connu : les représentations figurées y ont accoutumé les archéologues et les historiens de l'art. Toutefois, si la description en est aisée et le répertoire assez facile à établir, la question du sens en est étrangement négligée. Que peut bien signifier un tel geste, stéréotypé et récurrent, toujours en un même contexte ? Cette question semble avoir échappé à nos iconographes, par trop positivistes. Encore ne suffit-il pas de la poser...

Examinons néanmoins, à titre d'échantillons, trois images des plus célèbres : le Stamnos, signé Smikros, du musée de Bruxelles (fig. 1); un médaillon de Kylix des "Collections antiques» de Munich (fig. 2) et un fragment de scène latérale (sud) du sarcophage, dit «du plongeur» à Paestum (fig. 3).

Dans les trois cas, on retrouve le même geste, en une situation analogue, celle du banquet, et dans un semblable contexte musical. Or, ces images peuvent être lues, d'abord, d'une façon simplement dénotative. Le convive couché, la présence de l'aulète ou de l'aulétride, la situation de buveur, et d'auditeur, tout plaide en un premier temps pour une lecture "sympotique". Mais on risque de tomber assez vite dans la banalisation, ou la trivialité : du vin et de l'aulos à la saoûlerie et aux maux de tête... Sans doute, vaudrait-il mieux s'interroger sur le sens des deux principales composantes environnant nos images : la musique et le banquet.

D'abord, le Symposion n'est pas toujours, malgré son nom, une simple "beuverie collective». La référence aux récits homonymes de Platon et de Xénophon nous en fournit la preuve. De plus, le «banquet»lieu de plaisirs éminemment variables - peut aussi revêtir un statut métaphorique : image du bonheur, il servira parfois de terme comparant aux félicités de l'au-delà. Sa connotation variera, dès lors, de l'érotique au funèbre et comportera l'exigence au moins d'une double lecture. 
Comment expliquer autrement l'allusion de Socrate aux «banquets des bienheureux» au livre II de la République (363c) ? Comment lire autrement le décor à situation et destination funéraire du sarcophage de Paestum ? Même le très positiviste recueil Sympotica ${ }^{1}$ édité à Oxford par Oswyn Murray fait place, dans la contribution de John Boardman, au filigrane interprétatif d'un banquet dont l'instrument principal, la $\kappa \lambda i v \eta$, évoquerait également la célébration érotique des noces et l'exposition rituelle du défunt (lors de la prothesis). C'est de cette conjonction, d'ailleurs, que dépendra éventuellement l'interprétation symbolique d'une fonction sociale émargeant ainsi à l'aire du sacré2 2 .

De plus, le Symposion est toujours, de quelque manière, une célébration dionysiaque, dans les deux sens du terme : réunion de buveurs où le vin coule à flots, certes, mais où les propos du skolion peuvent toujours relayer le va-et-vient des coupes et nous emmener dans les plus hautes sphères de l'éros... Platon et Xénophon en témoigneraient à nouveau. On sait aussi que la cérémonie commence par une libation rituelle suivie d'un chant en l'honneur du dieu, péan ou dithyrambe. Ainsi amenés, les discours qui suivent peuvent ne pas être de simples propos de table mais participer des iepoi $\lambda$ óyor, de même que la boisson peut ne pas être simple beuverie érotique, mais évocation préparatoire d'un iepòs $\gamma \alpha \dot{\alpha} \mu o$ s. Sans oublier que les frontières du sacré et du profane étaient parfois chez les Grecs moins nettes que nous pourrions le croire. Si le temenos tranche l'espace et en délimite très exactement la double fonction, démarquant clairement le temple de son environnement, dans les actes de la vie quotidienne, on assiste souvent à un mixte de conduites qui, tout en étant utilitaires, portent la trace d'une sacralisation archaïque ou d'un rituel fossilisé. Ainsi, le Kottabos est peut-être la dégénérescence stochastique d'une offrande au dieu (de l'amour ?), pareillement, le symposiarque étendu sur le premier lit évoque, ou réalise, la présence du dieu Dionysos, de même que les convives représenteront ses comastes. Le gradian qui lestera la scène d'une plus ou moins grande sacralité reste évidemment à définir, selon les propos tenus, le désir de boire plus ou moins modéré et la volonté, parfois délibérée, de substituer à l'invective ou aux chansons gaillardes le chant profond.

La musique, quant à elle, ne doit pas davantage être réduite à sa fonction utilitaire ou décorative. Il ne s'agit pas d'une simple «musique de table» accompagnant un repas, mais aussi et surtout d'une pratique

1 A symposium on the Symposion, Clarendon Press, 1990.

2 Symposion Furniture, p. 122 sq. 
visant à charmer (au sens fort) quelques moments privilégiés de l'après-boire. Dès lors, ressouvenons-nous des théories musicales dont le sillage pythagoricien nous rappelle notamment le rôle psychagogique. Plus précisément lié au «banquet», l'aulos se signale par d'évidentes accointances dionysiaques. Cet instrument à anche, proche de notre hautbois, simple ou double, jouant toujours à l'unisson, représente en effet l'attrait fascinant et troublant des gammes "phrygiennes et lydiennes", les plus enharmoniques, modulant sur le quart de ton! La notion d'enthousiasme y est proverbialement liée, exprimant au niveau même du langage courant le souvenir d'une sacralisation de cette émotion violente. La musique de l'aulos est ainsi pourvoyeuse du raptus émotionnel où l'auditeur se croit "habité par le dieu", de même qu'elle réalise, comme nous le rappelle Aristote, l'allègement cathartique (Katharsis) de l'affection de l'âme (Pathos) ainsi provoquée ${ }^{3}$. Aristoxène, enfin, disciple de l'École, prêtera à l'ancien pythagorisme cet éclairant adage comparant à la médecine des corps la musique, "médecine des âmes»" 4 .

Rappelons ainsi que le son même de l'aulos évoque la présence de Dionysos ou annonce sa venue imminente ${ }^{5}$. Le vin qui tourne dans les coupes en signale également l'épiphanie. Toutefois, on veillera à ne pas oublier que Dionysos, maître de l'ivresse, est d'abord le dieu des sèves et de la fermentation printanière, avant d'être celui du vin, du moût et de l'automne 6 . La notion d'ivresse est donc relative elle aussi et peut s'entendre, même en l'absence de vin ou de tout agent physique à effet psychotrope, comme une affection de l'âme, analogue au vide intérieur du derviche ou aux photismes du Bodhisattva.

Les démarches mystiques (c'est-à-dire visant à l'union avec la divinité) ne sont pas plus réductibles aux seuls agents matériels en ce qui regarde les phénomènes d'ivresse que les impressions érotiques. En

3 VIII, 1341 b 33 - 1342 a 18. Cf. à ce propos mon Essai sur la Poétique d'Aristote, Paris, Vrin, 1975, p. 41, 68, 77 sq. Pour ce qui concerne les gammes et les réalités archéologiques ou instrumentales, cf. aussi F. DUYsInX, Musique et Poésie en Grèce antique, Bruxelles, F.P.G.L., 1988, p. 12 (aulos); 21 (gamme phrygienne et lydienne).

4 Diels-Kranz, Die Fragmente der Vorsokratiker, Berlin, $1961^{10}$, t. I, p. 768, 19 21.

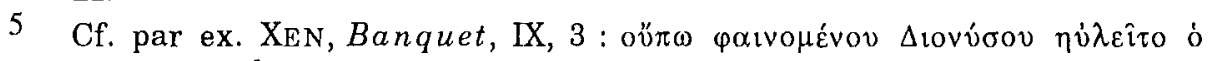
$\beta \alpha \kappa \chi \varepsilon \hat{\imath o \zeta ~} \dot{p} \theta \mu$ óç.

6 Cf. la belle monographie de W. OTTO, Dionysos, le mythe et le culte (trad. P. Levy), Paris, Mercure de France, 1969, p. 169 sq. et mes Etudes grecques, Bruxelles-Liège, Mardaga, 1990, p. 43 sq. 
tout cas, que le son de l'instrument (flûte ou hautbois) remplisse le cœur d'allégresse, nul ne l'aura mieux dit que Théognis :

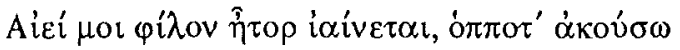

$\alpha \hat{\jmath} \lambda \hat{\omega} v \varphi \theta \varepsilon \gamma \gamma \circ \mu \varepsilon \dot{\varepsilon} v \omega \nu$ i $\mu \mu \varepsilon \rho o ́ \varepsilon \sigma \sigma \alpha \nu$ ö $\alpha \alpha$.

Toujours mon cœur bondit lorsque j'entends un son

De flûte au charme irrésistible.

(Élégies, I, 531-532)

Venons-en maintenant aux images, que nous sommes désormais mieux préparés à regarder et à tenter d'expliquer. La triple redite du même mouvement, confronté à la musique et au banquet, semble relever, par son aspect stéréotypé, d'une sorte de rituel ou de gestuelle dont le sens premier nous échappe, mais qui semble traditionnellement fixé. Le buste incliné et la tête projetée vers l'arrière, yeux grands ouverts, suggèrent à la fois la concentration et l'abandon, sous l'effet de l'audition musicale. Le mouvement pourrait rappeler le traditionnel geste ménadique projetant violemment la tête vers l'arrière, cou fléchi. Les sectatrices du dieu sont ainsi dites $\dot{\rho} \iota \psi \alpha v \chi \varepsilon ́ v \varepsilon \varsigma$ dans un fragment pindarique notamment ${ }^{7}$ et souvent représentées de la sorte, thyrse brandi, dans les scènes figurées. Mais notre mouvement paraît plus calme, du moins sous l'aboutissement iconographique qui nous en est présenté. L'auditeur bien concentré semble absorbé par la musique, comme ravi en extase où, étymologiquement parlant, l'âme semble quitter le corps... On pourrait croire aussi que le personnage chante, bien que souvent le geste qui aide à "pousser la note» soit plutôt, tête ainsi projetée vers l'arrière, celui de la main portée à la base du cou. La bouche entr'ouverte de l'auditeur de la fresque de Paestum peut également signifier qu'il s'abandonne à l'émotion. Rien n'impose de penser qu'il chante. Quant aux mots ov́ $\delta v ́ v \alpha \mu \alpha_{l}$ inscrits en légende sur le médaillon de Munich, rien ne nous contraint à les relier au texte du poème de Théognis :

«je ne peux chanter...»

(Élégies, I, 939-940)

7 Fragment du second Dithyrambe ( $70 \mathrm{~b}, 13$, Bowra) aimablement communiqué par mon collègue et ami Emilio Suarez de la Torre. 
On pourrait lire aussi, au regard de la coupe, endormie dans la main gauche :

«je ne peux boire...»

Sur l'image de Bruxelles, enfin, l'auditeur est comme fasciné par l'aulétride Helikè, dont les doigts et l'instrument (un diaulos merveilleusement découpé) touchent presque le bras et le visage. Les yeux ouverts, regardant à la verticale, la tête ceinte de bandelettes, c'est de son avant-bras qu'il se prend le front, la main reste pendante au niveau de l'arrière de la tête. Cet Alcibiade-là semble attendre Marsyas ou Socrate, indifférent au charme de la joueuse ionienne au délicieux drapé... Je m'empresse d'ajouter que les autres auditeurs sont aussi indifférents à la présence de l'aulète. Visiblement hors d'eux-mêmes, sous l'effet phrygien et dionysiaque de la musique, c'est le cortège du dieu qu'ils attendent ou entendent. Ils sont ainsi à l'image des Corybantes...

Suivons quelques instants cette autre piste d'enthousiasme musical. En trois endroits du plus haut lyrisme, Platon fait en son œuvre allusion à ce phénomène du corybantisme. La référence aux personnages mythiques qui auraient couvert de leur musique les vagissements de Zeus enfant, servant ainsi Rhéa, son auguste mère crétoise, nous offre un analogon du thiase dionysiaque. Le contexte d'ivresse musicale et de sons de flûte (aulos) y est toujours associé et Platon en fait à chaque fois état. Or, ceux dont il parle sont «comme les Corybantes», ou mieux encore "comme ceux qui sont en proie au délire corybantique» ( $\kappa о \rho \nu \beta \alpha \vee \tau(\hat{0} \vee \tau \varepsilon \varsigma)$. Suivent des notations d'hallucinations auditives s'accompagnant d'un état proche de la possession : les personnages sont dits $\kappa \alpha \tau \varepsilon \chi o ́ \mu \varepsilon v o r$ et offrent tous les signes de l'état d'extase musicale que nous avons cru lire sur nos images.

Voici ces textes de Platon. D'abord à la fin du Criton, après l'émouvante prosopopée des Lois, Socrate dit, en un moment de grande émotion puisqu'il vient de prendre la décision de ne pas se dérober à la mort, que ces discours lui ont précisément fait le même effet «que ces sons d'aulos que croient entendre ceux qui subissent les transports

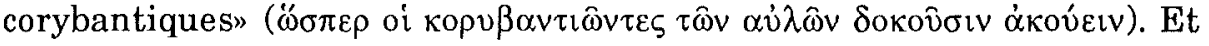

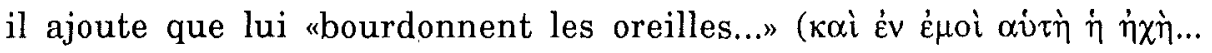
$\beta о \mu \beta \varepsilon \hat{\imath}, 54$ d).

Le deuxième passage, extrait de l'Ion, fait allusion à l'inspiration divine du rhapsode, soumis au génie homérique comme le poète créateur le sera à la Muse "de même que ceux qui subissent les transports corybantiques n'entendent que cette mélodie aiguë, qui doit être celle du 


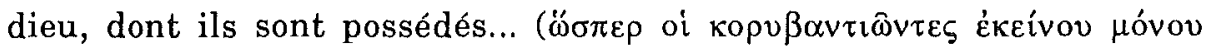

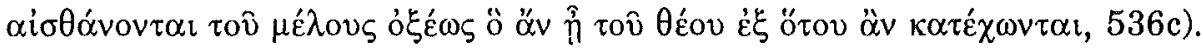

Le troisième extrait nous amène à la fin du Banquet, quand Alcibiade fait l'éloge de Socrate et le compare à un Silène ou à Marsyas, le célèbre aulète, disciple d'Olympos... Il ajoute alors que le charme de l'aulos, si exclusif et si divin par la qualité du raptus émotif qu'il nous

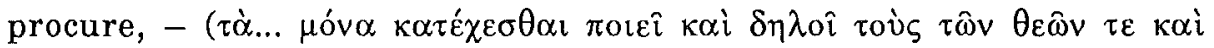

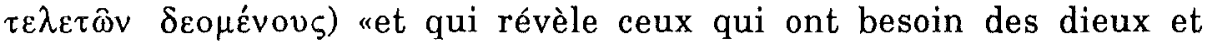
d'initiations", - Socrate est capable, à lui seul, de le produire sans aucun autre instrument que sa parole. Et Alcibiade d'ajouter : "quand je l'écoute, bien plus que chez ceux qui subissent les transports corybantiques, mon coeur bondit et les larmes me viennent sous l'effet de ses

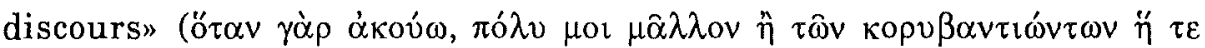

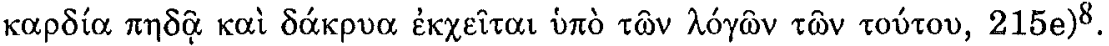

Cette triple affirmation, métaphorique, des effets proprement extatiques de l'aulos devrait éclairer notre petit problème de sémantique de l'image. Bien sûr, le texte n'est pas une «légende» comme le récit homérique peut quelquefois venir expliciter, par ekphrasis, telle représentation figurée du cadavre d'Hector traîné dans la poussière de Troie. Certains sujets, plus difficiles, comportent plus d'incertitudes.

Enfin, les allusions aux Corybantes nous permettront de nous tourner vers cette Crète où tout commence, où Zeus est né dans une Grotte de l'Ida, fils (et bientôt parèdre ?) de la Grande-Mère méditerranéenne des origines. C'est elle, Mère aussi des fauves et de la nature, qui se livre aux épiphanies végétales et chorégiques sur les sceaux et intailles du minoen moyen et récent. Que des phénomènes extatiques soient de son ressort et dépendent de ses «apparitions" semble chose admise. Que la musique (dont nous verrons défiler quelques instrumentistes sur les flancs du sarcophage peint d'Hagia-Triada) y jouent quelque rôle, est de l'ordre du plausible. Enfin, que le personnage, posté dans le coin latéral de telles scènes d'épiphanie ${ }^{9}$ ou représenté seul sous forme de statuette de

8 On notera, de même que chez Théognis le recours à des termes du vocabulaire amoureux, rappelant Sappho (fr. 2 Puech notamment) sans oublier le lien qui, de tout temps, rapproche l'érotique de la mystique.

9 Cf. notamment l'intaille reproduite par M.P. NILsson, Minoan-Mycenaean Religion and its Survival in Greek Religion, Lund, 1949², à la p. 353, fig. 162 où l'on voit trôner sur sa montagne, faite de pierres accumulées, une déesse des fauves héraldiquement encadrée de deux lionnes. 
bronze ${ }^{10}$, s'appliquant le poing droit sur le front, tronc légèrement arqué vers l'arrière, participe peu ou prou de notre thème iconographique, voilà qui pose une ultime question que, pour l'instant, nous laisserons au lecteur le soin d'apprécier.

En tout cas, la proximité initiatique et musicale des Courètes crétois, de l'aulos phrygien et des Bacchantes thébaines nous aura été redite par le plus jeune - et le plus tragique - des tragiques; écoutons Euripide dans la seconde antistrophe de la parodos des Bacchantes :

${ }^{\top} \Omega \theta \alpha \lambda \alpha \dot{\alpha} \mu \varepsilon u \mu \alpha$ Kovpq́-

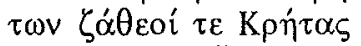

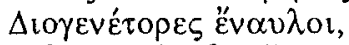

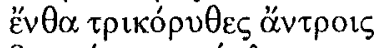

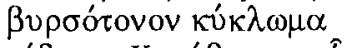

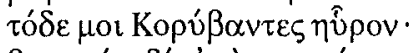

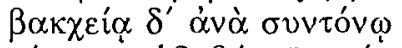

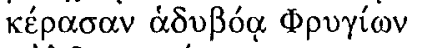
$\alpha \dot{\lambda} \lambda \omega \hat{\omega} v \pi v \dot{\mu} \mu \alpha \tau i$

(d'après l'éd. G. Murray)

Ô retraite des Courètes

ô grottes saintes de la Crète

où naquit Zeus, c'est là que découvrirent pour moi les Corybantes à triple aigrette ce cercle tendu de peau qu'ils joignirent fidèle au rythme des bacchantes au son délicieux des flûtes de Phrygie

Université de Liège

Pierre SOMVILLE

Philologie classique

Place du XX-Août, 32

B - 4000 LIĖGE

10 La plus célèbre est celle, trouvée à Tylissos, qui figure au musée d'Iraklion, mais on en trouve aussi de beaux exemplaires dans la collection Benaki ou au British Museum... 


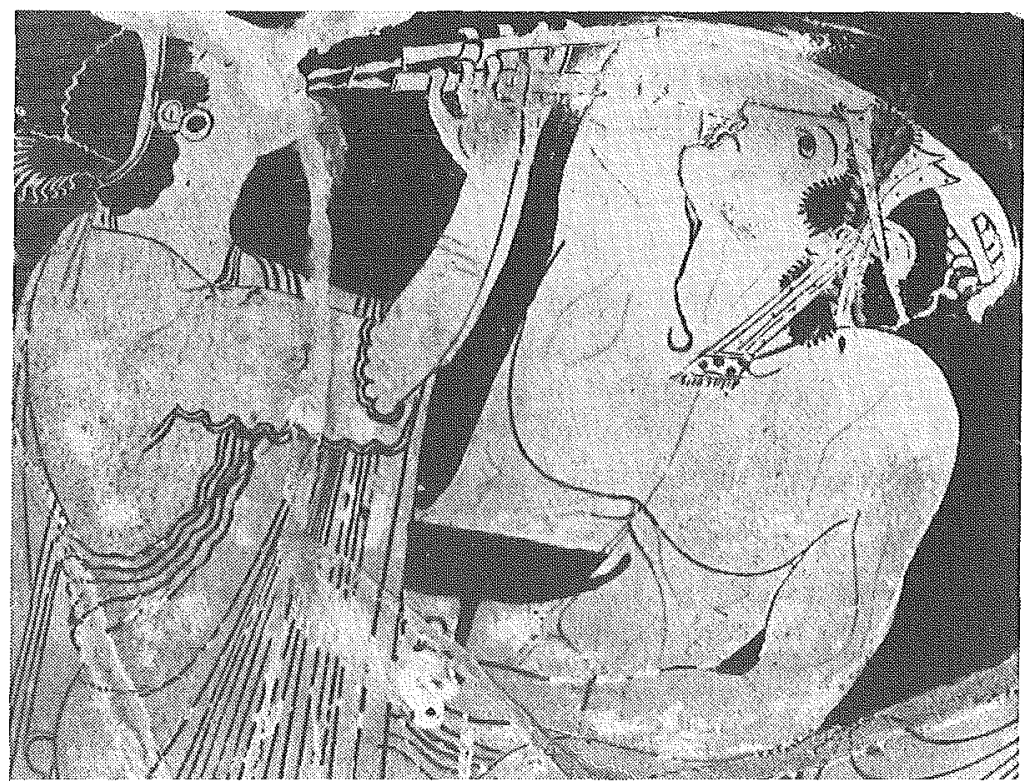

Fig. 1: Stamnos, signé Smikros, Ve s. av. J.-C., Bruxelles, Musée royaux d'art et d'histoire.

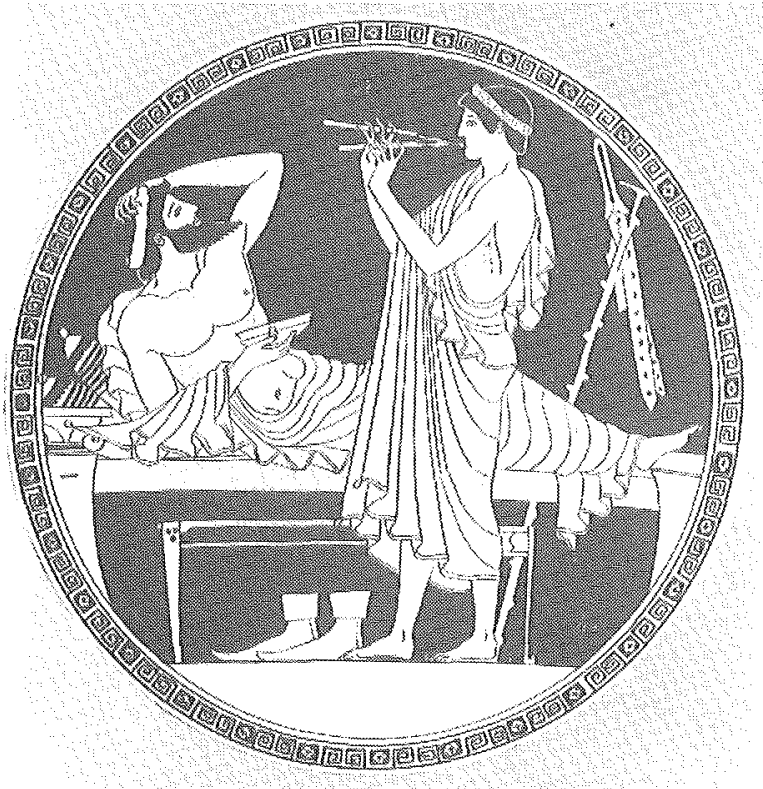

Fig. 2: Médaillon de coupe attribuée à Douris, Ve s. av. J.-C., Munich, Antike Sammlungen. 


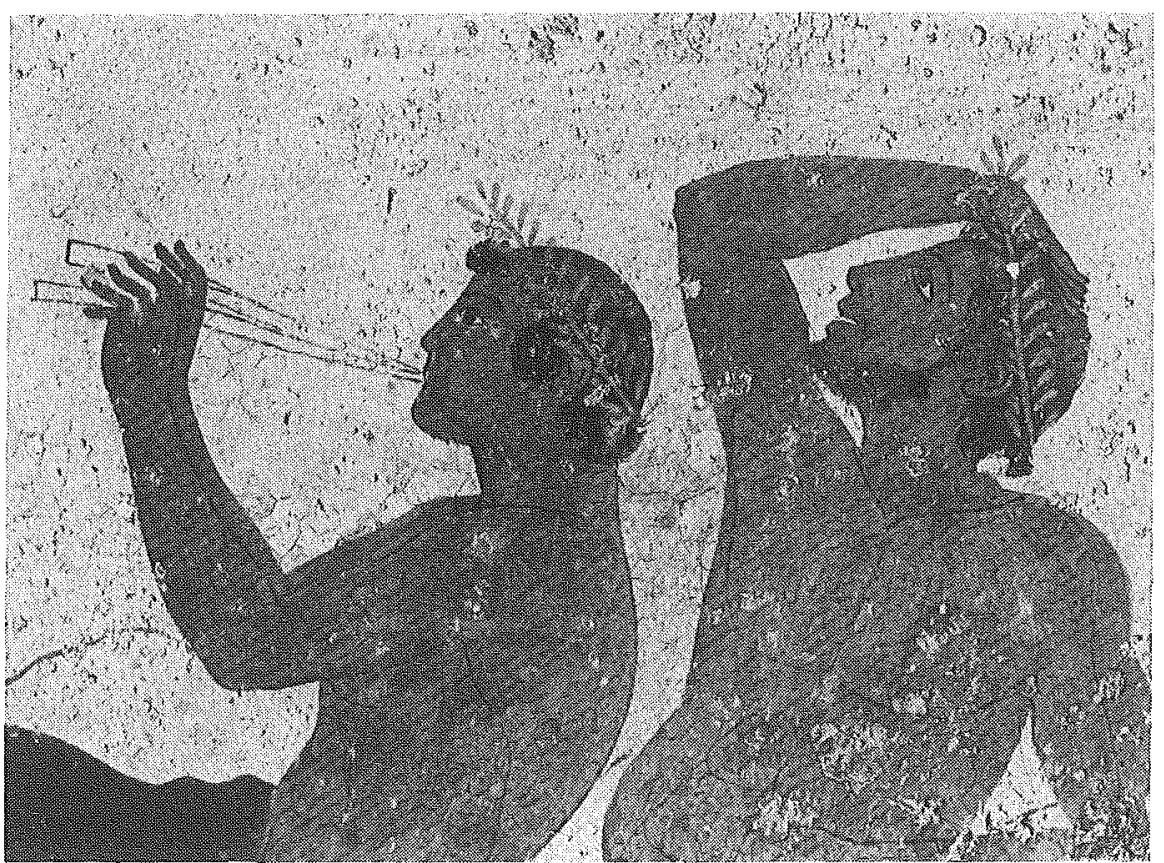

Fig. 3: Sarcophage peint, décor intérieur, long côté sud (fragm.), 480 av. J.-C., Musée de Paestum. 